\title{
Multiband and mass-anisotropy effects in the magnetoconductivity of a disordered electron gas
}

\author{
S. G. Magalhães \\ Departamento de Matemática, Universidade Federal de Santa Maria, 97119 Santa Maria, Rio Grande do Sul, Brazil \\ Alba Theumann \\ Instituto de Física, Universidade Federal do Rio Grande do Sul, Caixa Postal 15051, 91501-970 Porto Alegre, \\ Rio Grande do Sul, Brazil \\ (Received 28 July 1992)
}

\begin{abstract}
We investigate the magnetoconductance of a two-band electron system and a model with effectivemass anisotropy by means of the method based on the exact electron eigenstates in a magnetic field proposed formerly by us to study a single-band, isotropic system. We show the universality of the results, the details of the model being absorbed in properly defined effective diffusion constants, cyclotron frequencies, and lifetimes.
\end{abstract}

\section{INTRODUCTION}

In a previous publication, ${ }^{1}$ referred to as $\mathbf{I}$, we studied the magnetoconductivity of a one-band, isotropic, noninteracting electron gas in three dimensions by using the exact electron eigenstates in a magnetic field in place of the usual semiclassical approximation. ${ }^{2,3}$ The main result obtained was an anisotropic conductivity tensor for average fields with $\omega_{c} \tau \leq 1$, while the usual isotropic predictions in the semiclassical approximation were recovered in the weak-field limit $\omega_{c} \tau<<1$, where $\omega_{c}=e B / m_{0}$ is the electron cyclotron frequency and $\tau$ is the lifetime for elastic scattering.

In the present paper we investigate the universality of the results in I by applying the same method to the study of more realistic models in three dimensions. In previous work $^{4}$ the theory of Hikami, Larkin, and Nagaoka ${ }^{5}$ for two-dimensional systems was generalized to multiband electron models, and the results indicate that weak localization effects in these systems are indeed universal in terms of properly defined, effective diffusion constants and averaged lifetimes. Electron localization in anisotropic two-dimensional systems was studied in Ref. 6 in the absence of a magnetic field; there results for the magnetoconductivity are not presented. In this work the authors considered that anisotropic scattering is essential to obtain the correct observed behavior of the conductivity tensor, and within these assumptions they obtain an expression for the conductivity tensor where all anisotropy effects are absorbed in the definition of an anisotropic diffusion constant.

In order to test the universality of these results, we study in Sec. II the magnetoconductivity of a two-band system $^{7}$ of noninteracting electrons with the method of Ref. 1. We obtain that the diagonal components of the conductivity tensor consist of an isotropic universal term that depends only on an effective diffusion constant as predicted in Ref. 4, multiplied by an anisotropic contribution dependent on the band details. In the limit of weak fields we recover a universal, isotropic result as in I.

In Sec. III we analyze with the same method the mag- netoconductivity of a model with effective-mass anisotropy, ${ }^{6}$ although we consider only isotropic, $s$-like impurity scattering. We obtain that the magnetoconductivity tensor can be expressed in terms of anisotropic diffusion constants $D_{\mu}=\frac{2}{3}\left(\epsilon_{F} \tau / m_{\mu}\right)$ and of an effective cyclotron frequency $\omega_{c}=e B /\left(m_{x} m_{y}\right)^{1 / 2}$, where $B$ is the strength of the magnetic field in the $z$ direction, while $\epsilon_{F}, \tau$, and $m_{\mu}$ stand for the Fermi energy, inverse scattering lifetime, and effective mass in the $\mu$ direction, respectively. Although we consider only isotropic scattering, we obtain a universal contribution that scales as the conductivity in zero field of Ref. 6, multiplied by the anisotropic term obtained in $I$ for average field strengths.

\section{TWO-BAND ELECTRON MODEL}

We generalize the model of Ref. 7 to describe noninteracting electrons in two nonequivalent bands, in the presence of a constant magnetic field $B$ in the $z$ direction. The Hamiltonian is, in units where $\hbar=c=1$,

$$
\begin{gathered}
H=\sum_{\alpha, \beta}\left\{-\left(\delta_{\alpha \beta} / 2 m_{\alpha}\right) \int d \mathbf{r} \psi_{\alpha}^{+}(\mathbf{r}) \mathbf{D}_{r}^{2} \psi_{\alpha}(\mathbf{r})\right. \\
\left.+\int d \mathbf{r} \psi_{\alpha}^{+}(\mathbf{r}) V_{\alpha \beta}(\mathbf{r}) \psi_{\beta}(\mathbf{r})\right\},
\end{gathered}
$$

where $\alpha, \beta=1$ or 2 is the band index and $V_{\alpha \beta}$ is the scattering potential due to random impurities, with zero mean and variance:

$$
\begin{aligned}
\left\langle V_{\alpha \beta}(\mathbf{r}) V_{\alpha^{\prime} \beta^{\prime}}\left(\mathbf{r}^{\prime}\right)\right\rangle= & {\left[\Gamma_{0} \delta_{\alpha \beta} \delta_{\alpha^{\prime} \beta^{\prime}}\right.} \\
& \left.+\Gamma^{\prime}\left(1-\delta_{\alpha \beta}\right)\left(1-\delta_{\alpha^{\prime} \beta^{\prime}}\right)\right] \delta\left(\mathbf{r}-\mathbf{r}^{\prime}\right),
\end{aligned}
$$

where $\Gamma_{0}$ and $\Gamma^{\prime}$ are the strengths of intraband and interband scattering, respectively.

We also have in Eq. (1):

$$
\mathbf{D}_{r}=\nabla_{r}-i e \mathbf{A}_{0}(\mathbf{r}), \quad \mathbf{A}_{0}(\mathbf{r})=(-B y, 0,0) .
$$

The impurity-averaged quantities are obtained by standard diagrammatic methods ${ }^{8}$ and the averaged Green's 
function $G_{\alpha \beta}\left(\mathbf{r}, \mathbf{r}^{\prime} ; \epsilon\right)$ is given by the diagrams in Fig. 1, in the Born approximation. The off-diagonal components $G_{12} \approx O\left(\Gamma^{2}\right)$ and can be neglected; then we get for the diagonal ones, following the method in $I$,

$$
\begin{aligned}
G_{\alpha \alpha}\left(\mathbf{r}, \mathbf{r}^{\prime} ; \epsilon\right)= & \sum_{\lambda} \psi_{\lambda}^{+}(\mathbf{r}) \psi_{\lambda}\left(\mathbf{r}^{\prime}\right) G_{\alpha}\left(n, k_{z} ; \epsilon\right), \\
G_{\alpha}\left(n, k_{z} ; \epsilon\right)= & {\left[\omega_{\alpha}\left(n+\frac{1}{2}\right)+\left(1 / 2 m_{\alpha}\right) k_{z}^{2}-\epsilon_{F}\right.} \\
& \left.-\epsilon-\left(i / 2 \tau_{\alpha}\right) \operatorname{sgn}(\epsilon)\right]^{-1}
\end{aligned}
$$

where $\{\lambda\}=\left(n, k_{x}, k_{z}\right)$ indicate the set of Landau quantum numbers and $\psi_{\lambda}(\mathbf{r})$ is the wave function for an electron of mass $m_{\alpha}$ in a magnetic field. In the following we prefer to use the equivalent expression: ${ }^{1}$

$$
G_{\alpha}\left(\mathbf{r}, \mathbf{r}^{\prime}, \epsilon\right)=e^{i(e B / 2)\left(y+y^{\prime}\right)\left(x-x^{\prime}\right)} g_{\alpha}\left(\mathbf{r}-\mathbf{r}^{\prime}, \epsilon\right),
$$

with

$$
\begin{aligned}
g_{\alpha}(\mathbf{r}, \epsilon)=e B \sum_{n} \int & (d p / 2 \pi) e^{-i p z} e^{-e B\left(x^{2}+y^{2}\right) / 4} \\
& \times L_{n}\left[\frac{1}{2} e B\left(x^{2}+y^{2}\right)\right] G_{\alpha}(n, p, \epsilon) .
\end{aligned}
$$

In Eq. (4) we indicate by $\omega_{\alpha}=e B / m_{\alpha}$ the cyclotron frequency for electrons in each band, and $L_{n}(x)$ is a Laguerre polynomial. It is very important to remark at this point that the wave functions in Eqs. (4) and (6) depend only on the combination $\omega_{\alpha} m_{\alpha}=e B$ and they are in-

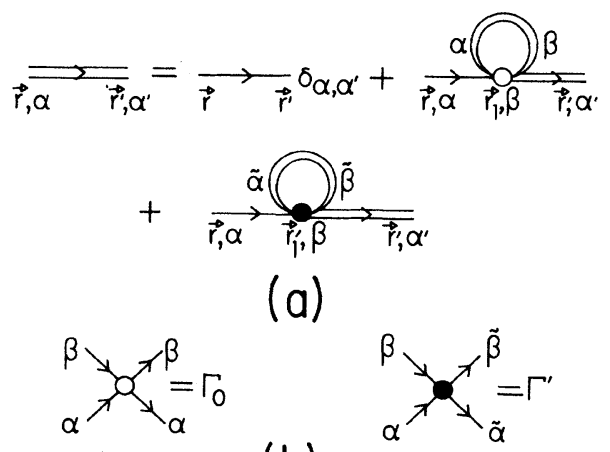

(b)

FIG. 1. (a) One-particle Green's function in the Born approximation for a two-band system in terms of the intraband and interband scattering potentials $\Gamma_{0}$ and $\Gamma^{\prime}$, shown in (b). Here $\alpha$ is the band index and $\widetilde{\alpha}=2$ or 1 if $\alpha=1$ or 2 .

dependent of the band index. This will allow us to solve the integral equation (10) below. The inverse lifetime in each band is given by

$$
1 / 2 \tau_{\alpha}=\Gamma_{0} N_{\alpha}+\Gamma^{\prime} N_{\bar{\alpha}},
$$

where $\widetilde{\alpha}=2(1)$ if $\alpha=1(2)$ and $N_{\alpha}=2 \pi \sqrt{2 \epsilon_{F}}\left(m_{\alpha}\right)^{3 / 2}$ is the density of states for the $\alpha$ band.

Standard linear-response theory ${ }^{8}$ gives for the diagonal components of the conductivity tensor:

$\sigma_{\mu \mu}(\mathbf{q}, \omega)=\frac{e^{2}}{4 \pi \omega} \int_{-\omega}^{0} d \epsilon \int d \mathbf{r}_{1}^{\prime} e^{i \mathbf{q} \cdot\left(\mathbf{r}^{\prime}{ }_{1}-\mathbf{r}\right)}\left[D_{r_{1}}^{\mu}-D_{r}^{\mu *}\right]_{r_{1}=r}\left[D_{r_{1}^{\prime}}^{\mu}-D_{r^{\prime}}^{\mu *}\right]_{r_{1}^{\prime}=r^{\prime}} \sum_{\alpha, \alpha^{\prime}} \frac{1}{m_{\alpha} m_{\alpha^{\prime}}} \Pi_{\alpha \alpha^{\prime}}\left(\mathbf{r}, \mathbf{r}^{\prime} ; \mathbf{r}_{1}^{\prime}, \mathbf{r}_{1}, \epsilon, \omega\right)$,

where the contributions of the multiply crossed diagrams to the diagonal polarizability $\Pi_{\alpha \alpha}$ are shown in Figs. 2 and 3. These processes are the same as those considered in Ref. 7 in the absence of a magnetic field. The off-diagonal II $\alpha \tilde{\alpha}$ does not present a diffusion pole and it can be discarded.

We get for $\Pi_{\alpha \alpha}$ from Fig. 2:

$$
\begin{aligned}
\Pi_{\alpha \alpha}\left(\mathbf{r}, \mathbf{r}^{\prime} ; \mathbf{r}_{1}^{\prime}, \mathbf{r}_{1}\right)= & G_{\alpha}^{+}\left(\mathbf{r}, r^{\prime}\right) G_{\alpha}^{-}\left(\mathbf{r}_{1}^{\prime} ; \mathbf{r}_{1}\right)+\Gamma_{0} \int d \mathbf{r}_{2} G_{\alpha}^{+}\left(\mathbf{r}, \mathbf{r}_{2}\right) G_{\alpha}^{+}\left(\mathbf{r}_{2} ; r^{\prime}\right) G_{\alpha}^{-}\left(\mathbf{r}_{1}^{\prime}, \mathbf{r}_{2}\right) G_{\alpha}^{-}\left(\mathbf{r}_{2}, \mathbf{r}_{1}\right) \\
& +\sum_{\alpha^{\prime} \alpha^{\prime \prime}} \int d \mathbf{r}_{3} d \mathbf{r}_{2} G_{\alpha}^{+}\left(\mathbf{r}, \mathbf{r}_{2}\right) G_{\alpha}^{-}\left(\mathbf{r}_{1}^{\prime}, \mathbf{r}_{2}\right) \Gamma_{\alpha \alpha^{\prime}} K_{\alpha^{\prime} \alpha^{\prime \prime}}\left(\mathbf{r}_{2}, \mathbf{r}_{3}\right) \Gamma_{\alpha^{\prime \prime} \alpha} G_{\alpha}^{+}\left(\mathbf{r}_{3}, \mathbf{r}^{\prime}\right) G_{\alpha}^{-}\left(\mathbf{r}_{3}, \mathbf{r}_{1}\right)
\end{aligned}
$$

where we introduced the notation $\Gamma_{11}=\Gamma_{22}=\Gamma_{0}$ and $\Gamma_{12}=\Gamma_{21}=\Gamma^{\prime}$ and $G_{\alpha}^{+}\left(\mathbf{r}, \mathbf{r}^{\prime}\right), \quad G_{\alpha}^{-}\left(\mathbf{r}, \mathbf{r}^{\prime}\right)$ stand for $G_{\alpha \alpha}\left(\mathbf{r}, \mathbf{r}^{\prime}, \omega+\epsilon\right), G_{\alpha \alpha}\left(\mathbf{r}, \mathbf{r}^{\prime}, \epsilon\right)$, respectively. The coupled integral equations for $K_{\alpha \alpha^{\prime}}\left(\mathbf{r}, \mathbf{r}^{\prime}\right)$ in Fig. 3 can be written in a compact $2 \times 2$ matrix form:

$\underline{K}\left(\mathbf{r}, \mathbf{r}^{\prime}\right)=\underline{K}^{0}\left(\mathbf{r}, \mathbf{r}^{\prime}\right)+\int d \mathbf{r}_{1} \underline{K}^{0}\left(\mathbf{r}, \mathbf{r}_{1}\right) \underline{\Gamma} \underline{K}\left(\mathbf{r}_{1}, \mathbf{r}^{\prime}\right)$,

where

$$
\underline{\Gamma}=\left[\begin{array}{cc}
\Gamma_{0} & \Gamma^{\prime} \\
\Gamma^{\prime} & \Gamma_{0}
\end{array}\right]
$$

and $\underline{K}_{0}$ is a diagonal matrix with elements

$$
\begin{aligned}
K_{\alpha \alpha}^{0}\left(\mathbf{r}, \mathbf{r}^{\prime}\right) & =G_{\alpha}^{+}\left(\mathbf{r}, \mathbf{r}^{\prime}\right) G_{\alpha}^{-}\left(\mathbf{r}, \mathbf{r}^{\prime}\right) \\
& =e^{i e B\left(y+y^{\prime}\right)\left(x-x^{\prime}\right)} g_{\alpha}\left(\mathbf{r}-\mathbf{r}^{\prime}, \omega+\epsilon\right) g_{\alpha}\left(\mathbf{r}-\mathbf{r}^{\prime}, \epsilon\right),
\end{aligned}
$$

where the last equality is obtained from Eq. (5). Just be- cause the exponential factor in Eq. (12) is independent of the band index, we can prove, as we did in $I$, that the solution of Eq. (10) can be written

$$
\underline{K}\left(\mathbf{r}, \mathbf{r}^{\prime}\right)=e^{i e B\left(x-x^{\prime}\right)\left(y+y^{\prime}\right)} \underline{\Lambda}\left(\mathbf{r}-\mathbf{r}^{\prime}\right),
$$

with $\underline{\Lambda}(\mathbf{r})$ satisfying the equation

$\underline{\Lambda}(\mathbf{r})=\underline{\Lambda}_{0}(\mathbf{r})+\int d \mathbf{r}_{1} \underline{\Lambda}_{0}\left(\mathbf{r}_{1}\right) \underline{\Gamma} \underline{\Lambda}\left(\mathbf{r}-\mathbf{r}_{1}\right) e^{i e B\left(x_{1} y-y_{1} x\right)}$,

where $\underline{\Lambda}_{0}$ is a diagonal matrix with elements

$$
\Lambda_{\alpha}^{0}(\mathbf{r})=g_{\alpha}(\mathbf{r} ; \omega+\epsilon) g_{\alpha}(\mathbf{r} ; \epsilon) .
$$

The solution of Eq. (14) is obtained as in I, first expanding to $O\left((e B)^{2}\right)$ and then solving for the Fourier transform $\underline{\Lambda}(\mathbf{k})$.

We obtain by Fourier transforming Eq. (15):

$$
\begin{aligned}
& \Lambda_{\alpha}^{0}(\mathbf{k})=2 \tau_{\alpha} N_{\alpha}\left[1-\theta_{\alpha}\right], \\
& \theta_{\alpha}=D_{\alpha}^{0} \tau_{\alpha} k_{z}^{2}+D_{\alpha}^{H} \tau_{\alpha} k_{1}^{2}-i \omega \tau_{\alpha},
\end{aligned}
$$




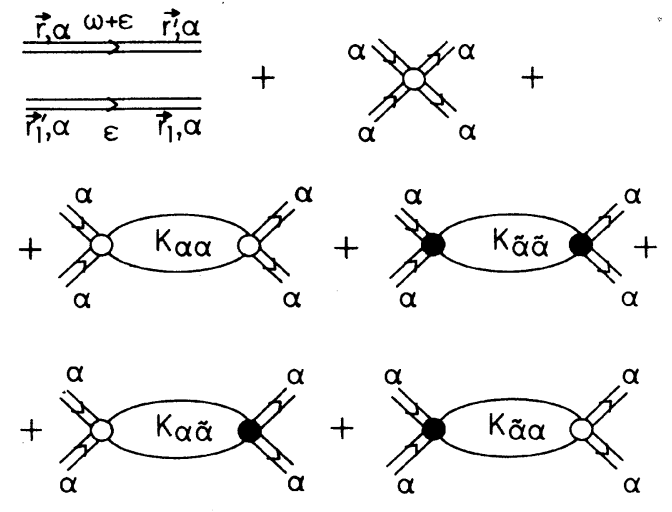

FIG. 2. Diagonal component of the polarizability as a function of the vertices $K_{\alpha \beta}$ in Fig. 3. The open and solid circles stand for $\Gamma_{0}$ and $\Gamma^{\prime}$, as in Fig. 1.

where

$D_{\alpha}^{0}=\frac{2}{3}\left(\epsilon_{F} \tau_{\alpha} / m_{\alpha}\right), \quad D_{\alpha}^{H}=D_{\alpha}^{0}\left[1+\left(\omega_{\alpha} \tau_{\alpha}\right)^{2}\right]^{-1}$

are the diffusion constants for each band and $\tau_{\alpha}$ was given in Eq. (7).

We obtain by expanding Eq. (14) to $O\left((e B)^{2}\right)$ that the Fourier-transformed matrix $\underline{\Lambda}(\mathbf{k})$ is given to this order by the equation

$$
\begin{aligned}
\underline{\Lambda}(\mathbf{k})=\underline{\bar{\Lambda}}(\mathbf{k})\left\{1-\frac{(e B)^{2}}{2} \underline{\Lambda}_{0}^{-1}[\right. & \frac{\partial^{2} \underline{\Lambda}_{0}}{\partial k_{x}^{2}} \underline{\Gamma} \frac{\partial^{2} \underline{\Lambda}}{\partial k_{y}^{2}}+\frac{\partial^{2} \underline{\Lambda}_{0}}{\partial k_{y}^{2}} \underline{\Gamma} \frac{\partial^{2} \underline{\Lambda}}{\partial k_{x}^{2}} \\
& \left.\left.-2 \frac{\partial^{2} \underline{\Lambda}_{0}}{\partial k_{x} \partial k_{y}} \Gamma \frac{\partial^{2} \underline{\Lambda}}{\partial k_{y} \partial k_{y}}\right]\right\},
\end{aligned}
$$

where, keeping only the leading terms in $\theta_{\alpha}$ in the denominator,

$$
\begin{gathered}
\underline{\bar{\Lambda}}(\mathbf{k})=\left[1-\underline{\Lambda}_{0} \underline{\Gamma}\right]^{-1} \underline{\Lambda}_{0}=\left[\left(\theta_{1} N_{1} / 2 \tau_{1}\right)+\left(\theta_{2} N_{2} / 2 \tau_{2}\right)\right]^{-1} \underline{M}, \\
\underline{M}=\left[\begin{array}{cc}
N_{1}^{2} & N_{1} N_{2} \\
N_{1} N_{2} & N_{2}^{2}
\end{array}\right] .
\end{gathered}
$$

The rest of the calculation is straightforward but tedious. We obtain to $O\left((e B)^{2}\right)$ :

$$
\underline{\Lambda}(\mathbf{k})=\underline{M} \frac{1}{N(0)\left(\frac{1}{2} k_{2}^{2} D_{\mathrm{eff}}^{0}+\frac{1}{2} k_{\perp}^{2} D_{\mathrm{eff}}^{H}\right)^{2}+\left(e B D_{\mathrm{eff}}^{0}\right)^{2}},
$$

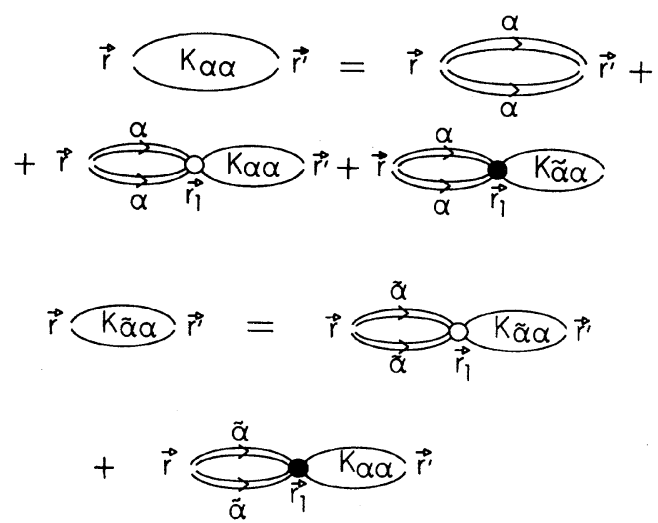

FIG. 3. Coupled integral equations for the vertex functions $K_{\alpha \alpha}$ and $K_{\alpha \tilde{\alpha}}$ with the same notation as in Figs. 2 and 1.

in terms of the effective diffusion constant

$$
\begin{aligned}
& D_{\text {eff }}^{0}=[1 / N(0)]\left[N_{1} D_{1}^{0}+N_{2} D_{2}^{0}\right], \\
& D_{\text {eff }}^{H}=\left[\frac{N_{1} D_{1}^{0}}{1+\left(\omega_{1} \tau_{1}\right)^{2}}+\frac{N_{2} D_{2}^{0}}{1+\left(\omega_{2} \tau_{2}\right)^{2}}\right] \frac{1}{N(0)},
\end{aligned}
$$

and the density of states $N(0)=N_{1}+N_{2}$.

The result obtained for $\underline{\Delta}(k)$ in Eq. (22) coincides with the corresponding expression in the single-band model calculated in I, the only difference being the dependence on the effective diffusion constant in Eq. (23).

By combining Eq. (22) with Eqs. (13) and (9), the calculation of the conductivity in Eq. (8) proceeds along identical lines as in $I$. To calculate $\Pi_{\alpha \alpha}$ in Eq. (9), we need

$$
\begin{aligned}
Q_{\alpha \alpha}(\mathbf{k})= & {[\underline{\Gamma} \underline{\Lambda}(\mathbf{k}) \underline{\Gamma}]_{\alpha \alpha} } \\
= & {\left[1 /\left(2 \tau_{\alpha}\right)^{2}\right][1 / N(0)] } \\
& \times \frac{\frac{1}{2} k_{z}^{2} D_{\mathrm{eff}}^{0}+\frac{1}{2} k_{\perp}^{2} D_{\mathrm{eff}}^{H}}{\left[\frac{1}{2} k_{z}^{2} D_{\mathrm{eff}}^{0}+\frac{1}{2} k_{\perp}^{2} D_{\mathrm{eff}}^{H}\right]^{2}+\left(e B D_{\mathrm{eff}}^{0}\right)^{2}},
\end{aligned}
$$

and we obtain for the diagonal components of the conductivity tensor:

$$
\sigma_{\mu \mu}=\sigma_{\mu \mu}^{0}+\sigma_{\mu \mu}^{\prime},
$$

where $\sigma_{\mu \mu}^{0}$ is the Lorentz resistivity and

$$
\begin{aligned}
& \sigma_{z z}^{\prime}=-\frac{e^{2}}{2 \pi^{2}} \sum_{\alpha} \frac{1}{m_{\alpha}^{2}} Q_{\alpha \alpha}(\mathbf{r}=0) \int_{-\infty}^{\infty} \frac{d p}{2 \pi} e B \sum_{n=0}^{\infty} p^{2}\left[G_{\alpha}^{+}(n, p) G_{\alpha}^{-}(n, p)\right]^{2}, \\
& \sigma_{\perp}^{\prime}=-\frac{e^{2}}{2 \pi^{2}} \sum_{\alpha} \frac{1}{m_{\alpha}^{2}} Q_{\alpha \alpha}(\mathbf{r}=0) \int_{-\infty}^{\infty} \frac{d p}{2 \pi}(e B)^{2} \sum_{n=0}^{\infty}\left\{\frac{n+1}{2}\left[G_{\alpha}^{+}(n, p) G_{\alpha}^{-}(n+1, p)\right]^{2}+\frac{n}{2}\left[G_{\alpha}^{+}(n, p) G_{\alpha}^{-}(n-1, p)\right]^{2}\right\},
\end{aligned}
$$

where $\sigma_{\perp}=\sigma_{x x}=\sigma_{y y}$ and the functions $G_{\alpha}^{ \pm}(n, p)=G_{\alpha}\left(n, p, \epsilon=0^{ \pm}\right)$were defined in Eq. (4).

The sums over Landau levels in Eqs. (26) and (27) were performed in I, with the result

$$
\sigma_{z z}^{\prime}=-\sqrt{3 / 2} \frac{e^{2}}{\pi^{4}} \frac{1}{N(0)} \frac{1}{\left(D_{\mathrm{eff}}\right)^{1 / 2} D_{\mathrm{eff}}^{H}}\left[I^{*}-\sqrt{e B D_{\mathrm{eff}}}\right] \sum_{\alpha}\left(\frac{m_{\alpha}}{\tau_{\alpha}}\right)^{2}\left(D_{\alpha} \tau_{\alpha}\right)^{3 / 2},
$$




$$
\begin{aligned}
\sigma_{\perp}^{\prime}=- & \sqrt{3 / 2} \frac{e^{2}}{\pi^{4}} \frac{1}{N(0)} \frac{1}{\left(D_{\mathrm{eff}}\right)^{1 / 2} D_{\mathrm{eff}}^{H}}\left[I^{*}-\sqrt{e B D_{\mathrm{eff}}}\right] \\
& \times \sum_{\alpha}\left[\frac{m_{\alpha}}{\tau_{\alpha}}\right]^{2}\left(D_{\alpha} \tau_{\alpha}\right)^{3 / 2}\left[1-6\left(\omega_{\alpha} \tau_{\alpha}\right)^{2}\right]
\end{aligned}
$$

where $I^{*}$ is an uninteresting, field-independent integration constant. In Eqs. (28) and (29) we may distinguish two different kinds of contributions: a universal isotropic term originating in the vertex function of Eq. (22), which reproduces the results of $I$ with $D_{0}$ replaced by $D_{\text {eff }}$, and a band-dependent, anisotropic factor. For sufficiently low fields such that $\omega_{\alpha} \tau_{\alpha} \ll<1$, we recover the isotropic magnetoconductance

$$
\sigma^{\prime}(e B)-\sigma^{\prime}(0) \approx \sqrt{e B D_{\mathrm{eff}}} \frac{e^{2}}{\pi^{4}} \frac{1}{N(0)} \sum_{\alpha} \frac{m_{\alpha}^{2}}{\sqrt{\tau_{\alpha}}}\left(\frac{D_{\alpha}}{D_{\mathrm{eff}}}\right)^{3 / 2} .
$$

\section{ANISOTROPIC SYSTEM}

We consider here a disordered electron system with anisotropic effective mass ${ }^{6} m_{\mu}$ in the $\mu$ direction, $\mu=x, y$, or $z$, in the presence of a magnetic field in the $z$ direction, with the Hamiltonian

$H=-\sum_{\mu} \frac{1}{2 m_{\mu}} \int d \mathbf{r} \psi^{+}(\mathbf{r}) D_{\mu}^{2} \psi(\mathbf{r})+\int d \mathbf{r} \psi^{+}(\mathbf{r}) V(\mathbf{r}) \psi(\mathbf{r})$,

where $D_{\mu}$ are the components of $\mathbf{D}_{r}$ in Eq. (3) and $V(\mathbf{r})$ is the isotropic scattering potential with zero mean and variance:

$$
\left\langle V(\mathbf{r}) V\left(\mathbf{r}^{\prime}\right)\right\rangle=\Gamma_{0} \delta\left(\mathbf{r}-\mathbf{r}^{\prime}\right) .
$$

The calculations follow along the lines of Sec. II and we just quote the results. The one-particle Green's function is given by an expression similar to Eq. (5):

$$
G\left(\mathbf{r}, \mathbf{r}^{\prime}, \boldsymbol{\epsilon}\right)=e^{i(e B / 2)\left(\rho_{x}-\rho_{x^{\prime}}\right)\left(\rho_{y}+\rho_{y^{\prime}}\right)} g\left(\boldsymbol{\rho}-\boldsymbol{\rho}^{\prime}, \boldsymbol{\epsilon}\right)
$$

in terms of the rescaled position vector $\rho$ with components

$$
\rho_{x}=\left(\frac{m_{x}}{m_{y}}\right)^{1 / 4} r_{x}, \quad \rho_{y}=\left(\frac{m_{y}}{m_{x}}\right)^{1 / 4} r_{y}, \quad \rho_{z}=z .
$$

The function $g(\rho, \epsilon)$ in Eq. (33) is given by the same expression as in Eq. (6) with

$$
\begin{aligned}
G(n, p, \epsilon)= & {\left[\omega_{c}\left(n+\frac{1}{2}\right)+\left(1 / 2 m_{z}\right) p^{2}-\epsilon_{F}\right.} \\
& -\epsilon-(i / 2 \tau) \operatorname{sgn}(\epsilon)]^{-1},
\end{aligned}
$$

where $\omega_{c}=e \boldsymbol{B} /\left(m_{x} m_{y}\right)^{1 / 2}$ and the inverse lifetime is as usual calculated with the diagrams in Fig. 1 for only one band. We obtain

$$
\begin{aligned}
& 1 / 2 \tau=\Gamma_{0} N_{a}, \\
& N_{a}=\left(m_{x} m_{y} m_{z}\right)^{1 / 2} N(0),
\end{aligned}
$$

and $N(0)=2 \pi \sqrt{2 \epsilon_{F}}$ is the density of states for electrons with isotropic effective mass equal to unity.

The diagonal components of the conductivity tensor are given by Eq. (8) for only one band, with the mass $m_{\alpha}$ replaced by the anisotropic $m_{\mu}$ and with the position vector $\mathbf{r}$ replaced by the rescaled vector $\rho$ in Eq. (34). The differential operators are also rescaled as

$$
\begin{aligned}
& D_{\rho}^{x}=\left(m_{x} / m_{y}\right)^{1 / 4}\left[\left(\partial / \partial \rho_{x}\right)-i e B \rho_{y}\right], \\
& D_{\rho}^{y}=\left(m_{y} / m_{x}\right)^{1 / 4}\left(\partial / \partial \rho_{y}\right), \quad D_{\rho}^{z}=\partial / \partial \rho_{z} .
\end{aligned}
$$

The calculation now follows identical steps as in $I$ or as in Sec. II for only one band in terms of the rescaled position variables of Eq. (34), because the exponential factor in Eqs. (12) and (13) remains invariant under this transformation of variables. The final result for the diagonal components of the conductivity tensor is

$\sigma_{\mu \mu}^{\prime}=-\frac{2 e^{2}}{\pi^{4}}\left(I^{*}-\sqrt{2 \omega_{c} \tau D_{0}}\right) \prod_{\nu}\left[\frac{1}{\left(D_{\nu} \tau\right)^{1 / 2}}\right]\left(D_{\mu} \tau\right)$

where

$$
D_{\mu}=\frac{2}{3}\left(\epsilon_{F} \tau / m_{\mu}\right)
$$

is the anisotropic diffusion constant and $D_{0}=\frac{2}{3} \epsilon_{F} \tau$ is the diffusion constant for isotropic unit mass. Although we consider only isotropic scattering potential, the expression of Eq. (39) could have been obtained phenomenologically from Ref. 6 by replacing the frequency $\omega$ by the cyclotron frequency $\omega_{c}$.

\section{CONCLUSIONS}

We have extended the method of I based on the use of the exact eigenstates of electrons in a magnetic field, to investigate localization effects in the magnetoconductance of more complicated systems in three dimensions, such as a two-band electron model and a model with effective-mass anisotropy. In both cases we obtained universal expressions in terms of suitably defined effective diffusion constants, as predicted by other authors. ${ }^{4,6}$

\section{ACKNOWLEDGMENTS}

We thank Dr. A. A. Gomes for calling our attention to this problem. This work was supported by Financiadora de Estudos e Projetos (FINEP) and Conselho Nacional de Desenvolvimento Científico e Tecnológico (CNPq).
${ }^{1}$ A. Theumann and S. G. Magalhães, Phys. Rev. B 39, 5798 (1989).

${ }^{2}$ P. A. Lee and T. V. Ramakrishnan, Rev. Mod. Phys. 57, 287 (1985).

${ }^{3}$ B. L. Altshuler and A. G. Aronov, in Electron-Electron Interactions in Disordered Systems, edited by A. L. Efros and M. Pollack (North-Holland, Amsterdam, 1985).
${ }^{4}$ D. Rainer and G. Bergmann, Phys. Rev. B 32, 3522 (1985).

${ }^{5}$ S. Hikami et al., Prog. Theor. Phys. 63, 707 (1980).

${ }^{6}$ D. Wölfle and R. N. Bhatt, Phys. Rev. B 30, 3542 (1984).

${ }^{7}$ H. Fukuyama, J. Phys. Soc. Jpn. 50, 3562 (1981).

${ }^{8}$ A. A. Abrikosov et al., Methods of Quantum Field Theory in Statistical Physics (Prentice-Hall, Englewood Cliffs, NJ, 1963), Chap. 7. 